\title{
Real-world Outcomes of First-line Afatinib in Patients with Non-small Cell Lung Cancer and Uncommon EGFR Mutations in South Korea
}

\section{Mi-Hyun Kim}

Pusan National University School of Medicine and biomedical Research Institute, Pusan National University Hospital

Chang Min Choi

Ulsan University Asan Medical Center

\section{Sung Yong Lee}

Korea University Guro Hospital

\section{Cheol Kyu Park}

Chonnam National University Hwasun Hospital

\section{Yoon Soo Chang}

Yonsei University Gangnam Severance Hospital

Kye Young Lee

Konkuk University Medical Center

\section{Seung Joon Kim}

Catholic University Seoul St. Mary's Hospital

Tae-Won Jang ( $\nabla$ jangtw22@hanmail.com )

Kosin University College of Medicine, Kosin University Gospel Hospital

\section{Sei Hoon Yang}

Wonkwang University Hospital

\section{Jeong Seon Ryu}

Inha University Hospital

Jeong Eun Lee

Chungnam National University

\section{Shin Yup Lee}

Kyungpook National University Chilgok Hospital

\section{Chan Kwon Park}

Catholic University Yeoudo St. Mary's Hospital

\section{Sang Hoon Lee}

Yonsei University Severance Hospital

\section{Seung Hun Jang}

Hallym University Sacred Heart Hospital 


\section{Seong Hoon Yoon}

Pusan National University Yangsan Hospital

\section{Research Article}

Keywords: non-small cell lung cancer, epidermal growth factor receptor (EGFR), exon 20 insertion, S768I

Posted Date: July 15th, 2021

DOl: https://doi.org/10.21203/rs.3.rs-697509/v1

License: (c) (1) This work is licensed under a Creative Commons Attribution 4.0 International License. Read Full License 


\section{Abstract}

Uncommon epidermal growth factor receptor (EGFR) mutations in non-small cell lung cancer (NSCLC) are heterogeneous and show variable prevalence and clinical responses to EGFR-tyrosine kinase inhibitors. We investigated the characteristics of uncommon EGFR mutations and the clinical efficacy of afatinib in patients. In this multicenter, retrospective study, we analyzed patients with NSCLC and uncommon EGFR mutations; these were categorized according to their incidence: (1) major uncommon mutations (G719X and L861Q), (2) compound mutations, and (3) minor uncommon mutations (exon 20 insertion, S768I, and de novo T790M). Sixty-four patients $(9.1 \%, 64 / 703)$ with uncommon EGFR mutations were identified in 16 South Korean institutes. Afatinib demonstrated activity against major uncommon (median time of treatment [TOT]: 20.3 months, $95 \%$ Cl: 5.0-27.9; overall survival (OS): 30.6 months, $95 \%$ Cl: $33.5-34.0$ ) and compound mutations (median TOT: 11.9 months, 95\% Cl: 3.6-9.6; OS: 29.1 months, $95 \%$ Cl: $12.1-$ 65.4). Minor uncommon mutations showed unfavorable responses to afatinib (median TOT: 3.8 months, $95 \%$ Cl: $2.1-4.2$; OS: 8.5 months, $95 \%$ Cl: $4.7-18.2)$. S768I mutation was present in 14 patients $(1.99 \%$, 14/703). Median TOT and OS were not significantly different between S768I and resistant exon 20 mutations. Afatinib is active in patients with NSCLC harboring major uncommon and compound EGFR mutations.

\section{Introduction}

Epidermal growth factor receptor (EGFR)-tyrosine kinase inhibitors (TKIs) are the first-line standard of care for patients with locally advanced or metastatic non-small-cell lung cancer (NSCLC) harboring EGFR mutations ${ }^{1}$. The most common type of EGFR mutation is exon 19 deletions, followed by the L858R point mutation in exon 21 , which account for $75-80 \%$ of patients with EGFR mutation-positive NSCLC ${ }^{2,3}$. They are conventionally referred to as common EGFR-TKI sensitive mutations based on numerous clinical trials ${ }^{4}$. However, other uncommon mutations represent a highly heterogeneous group and their clinical significance remains unclear.

Approximately $7-23 \%$ of NSCLC tumors harbor uncommon EGFR mutations ${ }^{5-12}$. The uncommon EGFR mutations include the primary drug resistance mutation T790M, exon 20 insertions, substitution mutations of L861Q, G719X, and S768I, and their coexisting mutations ${ }^{13}$. Double or multiple independent mutations of the EGFR-tyrosine kinase domain, termed compound mutations, account for up to $25 \%$ of all uncommon EGFR mutation-positive tumors ${ }^{14}$.

The clinical characteristics and most effective treatment strategies for many uncommon EGFR mutations are unclear. This uncertainty reflects the fact that they are rare and have been excluded from most clinical trials. In patients with uncommon EGFR mutations treated with first-generation EGFR-TKIs (gefitinib/erlotinib), the objective response rate (ORR) is approximately $40-50 \%$, and the median progression-free survival (PFS) is approximately 6-7.7 months ${ }^{15}$. A few studies have suggested that platinum-based cytotoxic chemotherapy or immunotherapy is preferable to EGFR-TKIs in some patients 
16-19. Recently, several studies have demonstrated the activity of a second-generation EGFR-TKI, afatinib, in patients with uncommon EGFR mutations. The Lux-Lung trials showed that the most prevalent uncommon mutations, G719X, L861Q, and S768I, appear to be sensitive to afatinib ${ }^{12}$. Additionally, a well-designed pooled analysis demonstrated the clinical activity of afatinib for the treatment of tumors harboring several uncommon EGFR mutation categories, especially certain exon 20 insertions, that confer resistance to EGFR-TKIs ${ }^{4}$. However, other studies have shown highly heterogeneous results of EGFR-TKIs against uncommon EGFR mutations.

Therefore, in this study, we aimed to evaluate the clinical characteristics of uncommon EGFR mutations and the real-world efficacy of first-line afatinib in a multicenter dataset of Korean patients with uncommon EGFR-positive advanced-stage NSCLC.

\section{Results}

\section{Patients}

In total, 703 patients with EGFR-mutated advanced-stage NSCLC were screened, and 64 (9.1\%) were analyzed in this study. Patient characteristics were not significantly different between patients with common EGFR mutations and those with uncommon EGFR mutations (data not shown). The characteristics of patients with uncommon EGFR mutations are shown in Table 1. The median age was 64 years (range, $32-84$ years), and 28 patients (44\%) were men. Thirty-eight patients (59\%) were never smokers, and $61(95 \%)$ had adenocarcinomas. 
Table 1

Clinical characteristics of non-small cell lung cancer patients with uncommon EGFR mutations. Values are presented as numbers (percentages), unless otherwise stated. ${ }^{a}$ Chi-square test: ECOG PS, Eastern Cooperative Oncology Group performance status.

\begin{tabular}{|c|c|c|c|c|c|}
\hline Characteristics & $\begin{array}{l}\text { Total } \\
(n=64)\end{array}$ & $\begin{array}{l}\text { Major } \\
(n=33)\end{array}$ & $\begin{array}{l}\text { Compound } \\
(n=23)\end{array}$ & $\begin{array}{l}\text { Minor } \\
(n=8)\end{array}$ & $\begin{array}{l}p \\
\text { value }^{\mathrm{a}}\end{array}$ \\
\hline $\begin{array}{l}\text { Age } \\
\text { (median, range) }\end{array}$ & $\begin{array}{l}66(32- \\
83)\end{array}$ & $\begin{array}{l}63(34- \\
83)\end{array}$ & $\begin{array}{l}67(32- \\
81)\end{array}$ & $\begin{array}{l}77(53- \\
81)\end{array}$ & 0.156 \\
\hline Male & $28(44)$ & $14(42)$ & $12(52)$ & $2(25)$ & 0.401 \\
\hline Smoking & $38(59)$ & $20(61)$ & $12(52)$ & $6(75)$ & 0.516 \\
\hline Never & $26(41)$ & $13(39)$ & $11(48)$ & $2(25)$ & \\
\hline \multicolumn{6}{|l|}{ Ever } \\
\hline ECOG PS & $58(92)$ & $29(88)$ & $22(96)$ & $7(100)$ & 0.407 \\
\hline $\begin{array}{l}0 \text { and } 1 \\
\geq 2\end{array}$ & $5(8)$ & $4(12)$ & $1(4)$ & $0(0)$ & \\
\hline Stage & $35(55)$ & $17(52)$ & $16(70)$ & $2(25)$ & 0.081 \\
\hline 3 & $29(45)$ & $16(48)$ & $7(30)$ & $6(75)$ & \\
\hline \multicolumn{6}{|l|}{4} \\
\hline Subtypes & $61(95)$ & $32(97)$ & $22(96)$ & $7(88)$ & 0.522 \\
\hline Adenocarcinoma & $3(5)$ & $1(3)$ & $1(4)$ & $1(12)$ & \\
\hline \multicolumn{6}{|l|}{ Others } \\
\hline $\begin{array}{l}\text { Median number of metastasis sites } \\
\text { (range) }\end{array}$ & $1.5(0-5)$ & $2(0-5)$ & $1(0-3)$ & $2(1-5)$ & 0.066 \\
\hline
\end{tabular}

The uncommon EGFR mutation categories are listed in Table 2. Overall, 33 patients (51\%) included in the analysis had a major uncommon mutation (G719X and L861Q), 23 (36\%) had a compound mutation, 4 (6\%) had an exon 20 insertion, 3 (5\%) had an S768I mutation, and 1 patient (2\%) had a T790M mutation. 
Table 2

EGFR mutation status of non-small cell lung cancer patients with uncommon EGFR mutations. Values are presented as numbers (percentages). ${ }^{a}$ sensitive mutations included EGFR 19 deletion and L858R mutation.

\begin{tabular}{|ll|}
\hline Mutation category & $\mathbf{N}(\%)$ \\
\hline Major uncommon mutation & $33(51)$ \\
G719X & $19(30)$ \\
L861Q & $14(21)$ \\
\hline Compound & $23(36)$ \\
G719X + S768I & $11(17)$ \\
G719X + L861Q & $4(6)$ \\
T790M + sensitive mutations & a \\
T790M + G719X & $4(6)$ \\
19deletion + 20insertion & $2(3)$ \\
19deletion + L861Q & $1(2)$ \\
\hline Exon 20 insertion & $1(2)$ \\
\hline S768I & $4(6)$ \\
\hline T790M & $3(5)$ \\
\hline
\end{tabular}

\section{Efficacy to afatinib in patients with uncommon EGFR mutations}

After receiving afatinib, patients with uncommon mutations exhibited a significantly inferior ORR (48.4\% vs. $67.1 \% ; p=0.003$ ), time of treatment (TOT) (median: 16.0 months vs. 24.3 months; $p=0.051$ ), and overall survival (OS) (median: 30.9 months vs. 50.9 months; $p=0.011$ ) than patients with common mutations.

Table 3 lists the ORRs of patients with the uncommon EGFR mutation subtype. The ORR was higher in patients with compound mutations $(\mathrm{ORR}=56.5 \%)$ and major uncommon mutations $(\mathrm{ORR}=46.9 \%)$ than in those with minor uncommon mutations $(\mathrm{ORR}=16.7 \%)$. 
Table 3

Tumor response to afatinib in non-small cell lung cancer patients with uncommon EGFR mutations. Values are presented as numbers and percentages. CR, complete response; PR, partial response; SD, stable disease; PD, progressive disease; NA, not-available; ORR, objective response rate; DCR, disease control rate.

\begin{tabular}{|llllllll|}
\hline Mutation category & \multicolumn{3}{l}{ Objective response } & \multicolumn{3}{c}{ ORR, \% } & DCR, \\
\cline { 2 - 6 } & CR, $\mathbf{n}$ & PR, $\mathbf{n}$ & SD, $\mathbf{n}$ & PD, $\mathbf{n}$ & NA, $\mathbf{n}$ & & \\
\hline Major uncommon mutations & 0 & 15 & 13 & 4 & 1 & 46.9 & 87.5 \\
G719X & & 9 & 8 & 2 & 0 & 47.3 & \\
L861Q & & 6 & 5 & 2 & 1 & 46.1 & \\
Compound mutations & 0 & 13 & 7 & 3 & 1 & 56.5 & 87.0 \\
Minor uncommon mutations & 0 & 1 & 3 & 2 & 2 & 16.7 & 66.7 \\
S768I & & 1 & 0 & 1 & 1 & & \\
Exon 20 insertion & & 0 & 3 & 1 & 0 & & \\
de novo T790M & 0 & 0 & 0 & 1 & & \\
\hline
\end{tabular}

The median TOT of all patients with uncommon mutations was 16.0 months (95\% confidence interval [CI]: 6.2-15.3). The major uncommon mutation group showed the longest TOT (median: 20.3 months, $95 \% \mathrm{Cl}: 5.0-27.9)$, followed by compound mutation (12.3 months, $95 \% \mathrm{Cl}: 3.9-9.9)$ and minor uncommon mutation (3.8 months, 95\% Cl: 2.1-4.2) groups (Fig. 1).

OS differed significantly between patients within uncommon mutation categories. Patients with the major uncommon mutation and compound mutation showed significantly longer OS than those in the minor uncommon mutation group (median: 30.6 months, $95 \% \mathrm{Cl}$ : 33.5-34.0 and 29.1 months, $95 \% \mathrm{Cl}: 12.1-$ 65.4 vs 8.5 months, $95 \%$ Cl: $4.7-18.2 ; p=0.036$ ) (Fig. 2).

Patients with minor uncommon mutations and any other mutation showed significantly longer survival than those with single minor mutations (median TOT: 10.6 months vs. 3.8 months; $p=0.014$ and median OS 28.8 months vs. 8.5 months; $p=0.065$ ) (Fig. 3).

\section{Outcomes in patients with tumors harboring minor uncommon mutations}

We categorized exon 20 insertions and S768I and T790M mutations as minor uncommon mutations according to their incidence rates. Among these, the S768I mutation was present in 14 patients $(1.99 \%$, 14/703). Only three patients had an isolated S768I mutation, and 11 had a concurrent G719X mutation. The ORR and disease control rate (DCR) were $58.3 \%$ and $83.3 \%$ for patients with the S768I mutation, respectively. The median TOT for patients with the S768I mutation was 8.2 months (95\% Cl: $5.3-7.3)$, which was not significantly different to that for patients with primary resistance mutations (exon 20 
insertions and T790M substitution) (8.7: months, $95 \% \mathrm{Cl}: 0.2-7.4 ; p=0.804)$. Moreover, the median OS was not significantly different between patients with $\mathrm{S768I}$ mutations and those with primary resistance mutations (22.9 months vs. 26.9 months; $p=0.728)$.

\section{Discussion}

This study showed the mutation patterns of NSCLC harboring uncommon EGFR mutations and the realworld efficacy of afatinib in Korean patients. Among 703 patients with advanced-stage NSCLC, we identified 64 cases of uncommon mutations, corresponding to a prevalence of $9.1 \%$. To the best of our knowledge, the current multicenter registry constitutes the largest study from Korea to report the clinical outcomes of patients with NSCLC and uncommon mutations using first-line afatinib. The most frequent uncommon mutations observed in our cohort were compound mutations (36\%), followed by G719X (30\%), L861Q (21\%), exon 20 mutations (12\%, exon 20 insertion [6\%], S768I [5\%], and de novo T790M [2\%]). The incidence rates of uncommon EGFR mutations vary significantly among ethnicities and countries. The incidence rate of exon 20 mutations in our study was lower than that reported in other studies. For example in a German cohort study, 4\% (23/573) had uncommon EGFR mutations, among which exon 20 mutations were the most frequent $(55 \%)^{20}$. Additionally, in an Indian cohort study, $6.58 \%$ $(83 / 1260)$ had uncommon mutations, among which exon 20 mutations were also the most common (exon 20 insertion, 19.3\%; T790M, 12\%; and S768I, 3.6\%) ${ }^{21}$.

The overall median TOT and OS of our cohort of patients treated with first-line afatinib were 16.0 months (95\% Cl: 6.2-15.3) and 30.9 months (95\% Cl: 22.3-45.2), respectively. Favorable efficacy of afatinib was observed in patients with major uncommon mutations (G719X and L861Q) and compound mutations. The median TOT and OS of patients using afatinib therapy for a major uncommon mutation were 20.3 months (95\% Cl: 5.0-27.9) and 30.6 months (95\% Cl: 33.5-34.0), respectively. Preclinical studies have shown that uncommon EGFR mutations, such as G719X and L861Q, are sensitive to first- and secondgeneration EGFR-TKIs ${ }^{22-24}$, and several clinical studies have reported that the median PFS can reach 7.7 months in patients with advanced NSCLC and uncommon EGFR mutations receiving EGFR-TKIs alone as first-line therapy ${ }^{15,25,26}$. These results are also comparable with the recently published data for afatinib. For instance, Yang et al. reported the efficacy of 315 patients with uncommon mutations who received first-line afatinib. In this analysis, the median time to treatment failure (TTF) of patients with major uncommon mutations was 10.8 months, suggesting a favorable outcome ${ }^{4}$. A major difference between our study and that by Yang et al. is that S768I was categorized as major uncommon mutation in Yang et al.

Compound EGFR mutations are present in up to a quarter of NSCLC tumors and are associated with poor prognosis ${ }^{3,27}$. However, the current study demonstrates that afatinib is active in most compound mutations, even in those with known resistance mutations, such as T790M and exon 20 insertion mutations. Median TOT (12.3 months) and OS (29.1 months) in patients with compound mutations were comparable with those in patients with major uncommon mutations. 
In this study, we showed that minor uncommon mutations, including exon 20 insertions, exon 20 S768I, and exon 20 T790M, were associated with an unfavorable response to afatinib. The median TOT and OS of patients using afatinib for these minor uncommon mutations were 3.8 months (95\% Cl: 2.1-4.2) and 8.5 months (95\% Cl: 4.7-18.2), respectively. These results are in line with those of Yang et al., who reported the TTF (median: 4.2 months) and ORR (24.3\%) in patients with an exon 20 insertion ${ }^{4}$, and suggest that afatinib shows inferior efficacy to that expected in patients treated with conventional platinum-based chemotherapy. Large-scale prospective clinical trials, such as IPASS, NEJ002, and WJTOG3405, showed consistent results that the median PFS of patients using conventional platinumbased chemotherapy is 5 to 6 months ${ }^{28-30}$. Additionally, several studies have claimed that EGFR-TKIs can be used as a subsequent therapy for patients with uncommon mutations, whereas platinum-based chemotherapy should be administered as a first-line treatment ${ }^{12,31,32}$. This supports our view that firstline platinum-based chemotherapy confers clinical benefits in patients with single exon 20 mutations. Recently, several agents targeting exon 20 insertions have been actively investigated. For example, a phase III study of mobocertinib (TAK-788), a novel, irreversible, small-molecule EGFR inhibitor specifically designed to target exon 20 insertion mutants, has been conducted in patients with advanced NSCLC harboring these mutations ${ }^{33}$. Preclinical data and phase $\mathrm{I} / \mathrm{Il}$ studies have reported that mobocertinib demonstrates antitumor activity with manageable toxicity in patients with advanced EGFR exon 20 insertion mutations ${ }^{33,34}$. Furthermore, the phase 1 CHRYSALIS trial (NCT02609776) showed that amivantamab, a bispecific antibody directed against EGF and MET receptors, induces an objective response in $40 \%$ of patients with previously treated NSCLC harboring EGFR exon 20 insertion mutations, with $47 \%$ maintaining their response for at least 6 months and $47 \%$ remaining on therapy after a median follow-up of over 9 months ${ }^{35}$. It was approved by the FDA in May 2021 as the first agent for adult patients with advanced NSCLC and EGFR exon 20 insertion mutations whose disease has progressed on or after platinum-based chemotherapy ${ }^{36}$.

We observed that first-line afatinib showed more favorable efficacy in patients harboring minor uncommon mutations with other mutations than in those harboring single minor uncommon mutations (median TOT: 10.6 months vs. 3.8 months; $p=0.014$ and median OS: 28.8 months vs. 8.5 months; $p=$ 0.065 ). This is consistent with studies from Taiwan (median PFS: 11.9 vs. 6.5 months; $p=0.010$ ) ${ }^{15}$ and China (median PFS: 9.3 vs 7.0 months; $p=0.4495$ ) ${ }^{17}$, in which the uncommon compound mutation group showed a relatively good response to EGFR-TKIs compared with the uncommon single-mutant group. Therefore, although single minor uncommon mutations do not necessarily confer sensitivity to afatinib, minor uncommon mutations with other EGFR mutations do not limit the efficacy of afatinib.

S768I mutations in EGFR exon 20 are rare $(1.99 \%, 14 / 703$ in our study) and are typically observed in combination with other EGFR mutations ${ }^{37}$. This mutation accounts for approximately $1 \%$ of all EGFR mutations ${ }^{38}$ and is usually categorized as a major uncommon mutation. Patients with this mutation reportedly have a favorable response to EGFR-TKIs ${ }^{39}$. However, in our study, we observed that patients with the $\mathbf{S 7 6 8 I}$ mutation showed a relatively unfavorable response to afatinib. Russo reported the case of an S768I mutation with a poor outcome after afatinib treatment. The rapid development of the T790M 
secondary mutation may be responsible for the low response to afatinib ${ }^{40}$. Unfortunately, we identified only three patients who underwent re-biopsy after progression to afatinib, and T790M development was not observed among these patients. As our sample size was small, the use of afatinib against S768I mutations requires confirmation in a larger scale study.

This study had several limitations. First, it was a retrospective study with an inevitable bias. Second, the detection rate of exon 20 mutations was very low; therefore, we could not conduct a subgroup analysis of each type of exon 20 mutation. We used the PNAClamp ${ }^{T M}$ EGFR Mutation Detection kit (PANAGENE, Inc., Daejeon, Korea) to detect EGFR mutations using real-time polymerase chain reaction (PCR). This technique is a highly sensitive method for detecting EGFR mutations but is restricted to several targetable mutations ${ }^{41}$. We believe that this might have contributed to the low incidence of exon 20 mutations. Byeon et al. reported that 27 of 1,419 patients with advanced NSCLC had an exon 20 insertion in Korea ${ }^{42}$. They used various techniques to detect EGFR mutations, including next-generation sequencing (NGS), which indicates that highly sensitive NGS techniques can expand the detection of targetable somatic mutations in EGFR. Third, TOT and OS might have been overestimated in the study owing to the small sample size. Despite these limitations, this study is the first multicenter study in South Korea to report the clinical outcomes of patients with NSCLC and uncommon mutations with using firstline afatinib based on real-world experience, and its applicability to real clinical practice, especially for Asian populations, could allow better patient management and improved survival outcomes in patients with uncommon EGFR mutations.

In conclusion, this study demonstrated the efficacy of first-line afatinib for the treatment of tumors harboring uncommon EGFR mutations, especially G719X, L816Q, and compound mutations. Although the incidence of S768I, exon 20 insertion, and de novo T790M mutations was low, patients with these mutations showed unfavorable responses to first-line afatinib and poor survival outcomes.

\section{Methods}

\section{Study design and participants}

Details of patients with tumors harboring uncommon mutations treated with first-line afatinib in the Realworld Experience of SEquential Treatment of afatinib followed by osimertinib (RESET) study, a retrospective multicenter observational study across 16 medical centers in South Korea, were collected. Briefly, eligible patients were aged 19 years or older with treatment-naïve, histologically or cytologically documented NSCLC that was American Joint Committee on Cancer (8th edition) stage 3B, 3C, 4A, or 4B. Key exclusion criteria were patients who were not treated with afatinib as first-line therapy. Electronic medical records from October 2014 to October 2019 were reviewed. The peptide nucleic acid-mediated real-time PCR clamping method was used to detect EGFR mutations in all medical centers.

Uncommon mutations were categorized into three groups according to their incidence rate: (1) major uncommon mutations (G719X and L861Q), (2) compound mutations, and (3) minor uncommon 
mutations (exon 20 insertion, S768I, and de novo T790M). One unusual case and cases with both 19 deletions and L858R mutations were excluded from the analysis.

\section{Clinical data collection and efficacy evaluations}

Baseline demographic characteristics (age, sex, and Eastern Cooperative Oncology Group performance status) were collected. Smoking status was categorized into never, former, and current smokers according to the classification of the National Health Interview Survey. The date of diagnosis, initiation of first-line afatinib, information on EGFR mutation (i.e., presence/absence and profile), number of metastatic organs, existence of specific organ metastasis, and dose modification of afatinib were recorded.

Efficacy outcomes included ORR, DCR, TOT, and OS. Tumor response was assessed using computed tomography scans, but decisions concerning the follow-up intervals or radiologic tools were entirely at the discretion of the physician. Response to afatinib was calculated according to Response Evaluation Criteria in Solid Tumors 1.1. TOT was defined as the time from the start of therapy to treatment discontinuation for any reason or death. This was chosen as a pragmatic endpoint suited to real-world studies ${ }^{4}$. OS was calculated from the date of therapy initiation to the date of death.

\section{Statistical analysis}

The patients' baseline characteristics are shown as descriptive data. Chi-squared and Fisher's exact tests were used to compare differences between categorical variables. TOT and OS were estimated using the Kaplan-Meier method, and differences in time distributions were compared using the log-rank test. The estimated median time (months) and $95 \% \mathrm{Cl}$ are presented. All p-values were two-sided, and statistical significance was set at $p<0.05$. All statistical analyses were performed using IBM SPSS Statistics for Windows (version 25.0; IBM Corp., Armonk, NY, USA).

\section{Ethics approval}

The study and protocol were approved by the Institutional Review Board (IRB) of Kosin University Gospel Hospital (IRB no. 2021-06-22) and Pusan National University Hospital (IRB no. 2107-001-104) and was conducted in accordance with the principles of the Declaration of Helsinki. Informed consent was waived by the IRB of Kosin University Gospel Hospital (IRB no. 2021-06-22) and Pusan National University Hospital (IRB no. 2107-001-104) because of the retrospective nature of the study and the analysis used anonymous clinical data.

\section{Data Availability}


The datasets generated and analysed during the current study are available from the corresponding author on reasonable request.

\section{Declarations}

\section{Acknowledgements}

The funding source had no role in the design, preparation, or submission of the manuscript. None of the authors received payments or services from a third party for the submitted work. We would like to thank Editage (www.editage.com) for English language editing.

\section{Author contributions}

Study concept and design: K.M., and J.TW. Acquisition of data: K.M., C.CM., L.SY., P.CK., C.YS., L.KY., K.SJ., L.JE., L.SY., P.CK., L.SH., J.SH., Y.SH., and J.TW. Analysis and interpretation of data: K.M., C.CM. and J.TW., Drafting of manuscript: K.M., and J.TW. Critical revision: C.CM., L.SY., P.CK., C.YS., L.KY., K.SJ., L.JE., L.SY., P.CK., L.SH., J.SH., Y.SH., and J.TW. All authors contributed to the approval of the submitted and final versions.

\section{Competing Interests Statement:}

The author(s) declare no competing interests.

\section{Additional information}

Correspondence and requests for materials should be addressed to J.TW.

\section{References}

1. National Comprehensive Cancer Network. (2021). Non-small cell lung cancer (version 5.2021). Retrieved from https://www.nccn.org/professionals/physician_gls/pdf/nscl.pdf (2021)

2. Kobayashi, S. et al. Compound EGFR mutations and response to EGFR tyrosine kinase inhibitors. $J$ Thorac Oncol, 8, 45-51 (2013).

3. Kim, E. Y. et al. g. Compound EGFR mutation is frequently detected with co-mutations of actionable genes and associated with poor clinical outcome in lung adenocarcinoma. Cancer Biol Ther, 17, 237-245 (2016).

4. Yang, J. C. H. et al. Afatinib for the Treatment of NSCLC Harboring Uncommon EGFR Mutations: A Database of 693 Cases. J Thorac Oncol, 15, 803-805 (2020). 
5. Keam, B. et al. Rare and complex mutations of epidermal growth factor receptor, and efficacy of tyrosine kinase inhibitor in patients with non-small cell lung cancer. Int J Clin Oncol, 19, 594-600 https://doi.org/10.1007/s10147-013-0602-1 (2014).

6. Kuiper, J. L. et al. Non-classic EGFR mutations in a cohort of Dutch EGFR-mutated NSCLC patients and outcomes following EGFR-TKI treatment. Br J Cancer, 115, 1504-1512 https://doi.org/10.1038/bjc.2016.372 (2016).

7. Shen, Y. C. et al. Comparing the effects of afatinib with gefitinib or Erlotinib in patients with advanced-stage lung adenocarcinoma harboring non-classical epidermal growth factor receptor mutations. Lung Cancer, 110, 56-62 https://doi.org/10.1016/j.lungcan.2017.06.007 (2017).

8. Kris, M. G. et al. Using multiplexed assays of oncogenic drivers in lung cancers to select targeted drugs. Jama, 311, 1998-2006 https://doi.org/10.1001/jama.2014.3741 (2014).

9. Beau-Faller, M. et al. Rare EGFR exon 18 and exon 20 mutations in non-small-cell lung cancer on 10 117 patients: a multicentre observational study by the French ERMETIC-IFCT network. Ann Oncol, 25, 126-131 https://doi.org/10.1093/annonc/mdt418 (2014).

10. Krawczyk, P. et al. Comparison of the effectiveness of erlotinib, gefitinib, and afatinib for treatment of non-small cell lung cancer in patients with common and rare EGFR gene mutations. Oncol Lett, 13, 4433-4444 https://doi.org/10.3892/ol.2017.5980 (2017).

11. Heigener, D. F. et al. Afatinib in Non-Small Cell Lung Cancer Harboring Uncommon EGFR Mutations Pretreated With Reversible EGFR Inhibitors. Oncologist, 20, 1167-1174 https://doi.org/10.1634/theoncologist.2015-0073 (2015).

12. Yang, J. C. et al. Clinical activity of afatinib in patients with advanced non-small-cell lung cancer harbouring uncommon EGFR mutations: a combined post-hoc analysis of LUX-Lung 2, LUX-Lung 3, and LUX-Lung 6. Lancet Oncol, 16, 830-838 https://doi.org/10.1016/s1470-2045(15)00026-1 (2015).

13. Baek, J. H. et al. Efficacy of EGFR tyrosine kinase inhibitors in patients with EGFR-mutated non-small cell lung cancer except both exon 19 deletion and exon 21 L858R: a retrospective analysis in Korea. Lung Cancer, 87, 148-154 https://doi.org/10.1016/j.lungcan.2014.11.013 (2015).

14. Syahruddin, E. et al. Uncommon EGFR mutations in cytological specimens of 1,874 newly diagnosed Indonesian lung cancer patients. Lung Cancer (Auckl), 9, 25-34 https://doi.org/10.2147/lctt.S154116 (2018).

15. Chiu, C. H. et al. Epidermal Growth Factor Receptor Tyrosine Kinase Inhibitor Treatment Response in Advanced Lung Adenocarcinomas with G719X/L861Q/S768I Mutations. J Thorac Oncol, 10, 793799 https://doi.org/10.1097/jto.0000000000000504 (2015).

16. Brindel, A. et al. Uncommon EGFR mutations in lung adenocarcinoma: features and response to tyrosine kinase inhibitors. J Thorac Dis, 12, 4643-4650 https://doi.org/10.21037/jtd-19-3790 (2020).

17. Li, H. et al. Efficacy and long-term survival of advanced lung adenocarcinoma patients with uncommon EGFR mutations treated with 1st generation EGFR-TKIs compared with chemotherapy as first-line therapy. Lung Cancer, 130, 42-49 https://doi.org/10.1016/j.lungcan.2019.02.001 (2019). 
18. Yamada, T. et al. Retrospective efficacy analysis of immune checkpoint inhibitors in patients with EGFR-mutated non-small cell lung cancer. Cancer Med, 8, 1521-1529 https://doi.org/10.1002/cam4.2037 (2019).

19. Socinski, M. A. et al. Atezolizumab for First-Line Treatment of Metastatic Nonsquamous NSCLC. $N$ Engl J Med, 378, 2288-2301 https://doi.org/10.1056/NEJMoa1716948 (2018).

20. Heigener, D. F. et al. Afatinib in Non-Small Cell Lung Cancer Harboring UncommonEGFRMutations Pretreated With Reversible EGFR Inhibitors. The Oncologist, 20, 1167-1174 https://doi.org/10.1634/theoncologist.2015-0073 (2015).

21. Kate, S. et al. Outcome of uncommon EGFR mutation positive newly diagnosed advanced non-small cell lung cancer patients: a single center retrospective analysis. Lung Cancer: Targets and Therapy, 10, 1-10 https://doi.org/10.2147/lctt.s181406 (2019).

22. Chen, Y. R. et al. Distinctive activation patterns in constitutively active and gefitinib-sensitive EGFR mutants. Oncogene, 25, 1205-1215 https:// doi.org/10.1038/sj.onc.1209159 (2006).

23. Kancha, R. K., Peschel, C. \& Duyster, J. The epidermal growth factor receptor-L861Q mutation increases kinase activity without leading to enhanced sensitivity toward epidermal growth factor receptor kinase inhibitors. J Thorac Oncol, 6, 387-392 https://doi.org/10.1097/JT0.0b013e3182021f3e (2011).

24. Jiang, J. et al. Epidermal growth factor-independent transformation of Ba/F3 cells with cancerderived epidermal growth factor receptor mutants induces gefitinib-sensitive cell cycle progression. Cancer Res, 65, 8968-8974 https://doi.org/10.1158/0008-5472.Can-05-1829 (2005).

25. Wu, J. Y. et al. Effectiveness of tyrosine kinase inhibitors on "uncommon" epidermal growth factor receptor mutations of unknown clinical significance in non-small cell lung cancer. Clin Cancer Res, 17, 3812-3821 https://doi.org/10.1158/1078-0432.Ccr-10-3408 (2011).

26. Zhang, Y. et al. Clinical characteristics and response to tyrosine kinase inhibitors of patients with non-small cell lung cancer harboring uncommon epidermal growth factor receptor mutations. Chin $J$ Cancer Res, 29, 18-24 https://doi.org/10.21147/j.issn.1000-9604.2017.01.03 (2017).

27. Kohsaka, S. et al. A method of high-throughput functional evaluation of EGFR gene variants of unknown significance in cancer. Sci Trans/ Med, 9, https://doi.org/10.1126/scitranslmed.aan6566 (2017).

28. Mok, T. S. et al. Gefitinib or Carboplatin-Paclitaxel in Pulmonary Adenocarcinoma. N Engl J Med, 361, 947-957 https://doi.org/10.1056/nejmoa0810699 (2009).

29. Maemondo, M. et al. Gefitinib or Chemotherapy for Non-Small-Cell Lung Cancer with Mutated EGFR. N Engl J Med, 362, 2380-2388 https://doi.org/10.1056/nejmoa0909530 (2010).

30. Mitsudomi, T. et al. Gefitinib versus cisplatin plus docetaxel in patients with non-small-cell lung cancer harbouring mutations of the epidermal growth factor receptor (WJTOG3405): an open label, randomised phase 3 trial. Lancet Oncol, 11, 121-128 https://doi.org/10.1016/s14702045(09)70364-x (2010). 
31. Shi, J. et al. Uncommon EGFR mutations in a cohort of Chinese NSCLC patients and outcomes of first-line EGFR-TKIs and platinum-based chemotherapy. Chin J Cancer Res, 29, 543-552 https://doi.org/10.21147/j.issn.1000-9604.2017.06.09 (2017).

32. Arrieta, O. et al. The impact of common and rare EGFR mutations in response to EGFR tyrosine kinase inhibitors and platinum-based chemotherapy in patients with non-small cell lung cancer. Lung Cancer, 87, 169-175 https://doi.org/10.1016/j.lungcan.2014.12.009 (2015).

33. Gonzalvez, F. et al. Mobocertinib (TAK-788): A Targeted Inhibitor of EGFR Exon 20 Insertion Mutants in Non-Small Cell Lung Cancer. Cancer Discov, https://doi.org/10.1158/2159-8290.Cd-20-1683 (2021).

34. Riely, G. J. et al. Activity and Safety of Mobocertinib (TAK-788) in Previously Treated Non-Small Cell Lung Cancer with EGFR Exon 20 Insertion Mutations from a Phase I/II Trial. Cancer Discov, https://doi.org/10.1158/2159-8290.Cd-20-1598 (2021).

35. Sabari, J. K. et al. in IASLC 2020 World Conference on Lung Cancer 28-31 (Singapre, 2021).

36. FDA grants accelerated approval to amivantamab-vmjw for metastatic non-small cell lung cancer, https://www.fda.gov/drugs/resources-information-approved-drugs/fda-grants-accelerated-approvalamivantamab-vmjw-metastatic-non-small-cell-lung-cancer (2021).

37. Leventakos, K. et al. S768I Mutation in EGFR in Patients with Lung Cancer. J Thorac Oncol, 11, 1798-1801 https://doi.org/10.1016/j.jtho.2016.05.007 (2016).

38. Arcila, M. E. et al. EGFR exon 20 insertion mutations in lung adenocarcinomas: prevalence, molecular heterogeneity, and clinicopathologic characteristics. Mol Cancer Ther, 12, 220-229 https://doi.org/10.1158/1535-7163.Mct-12-0620 (2013).

39. Zhu, X. et al. Response to Tyrosine Kinase Inhibitors in Lung Adenocarcinoma with the Rare Epidermal Growth Factor Receptor Mutation S768I: a Retrospective Analysis and Literature Review. Target Oncol, 12, 81-88 https://doi.org/10.1007/s11523-016-0455-4 (2017).

40. Russo, A., Franchina, T., Ricciardi, G. R. R. \& Adamo, V. Rapid Acquisition of T790M Mutation after Treatment with Afatinib in an NSCLC Patient Harboring EGFR Exon 20 S768I Mutation. $J$ Thorac Oncol, 12, e6-e8 https://doi.org/10.1016/j.jtho.2016.09.132 (2017).

41. Kim, H. J. et al. Detection and comparison of peptide nucleic acid-mediated real-time polymerase chain reaction clamping and direct gene sequencing for epidermal growth factor receptor mutations in patients with non-small cell lung cancer. Lung Cancer, 75, 321-325 https://doi.org/10.1016/j.lungcan.2011.08.005 (2012).

42. Byeon, S. et al. Clinical Outcomes of EGFR Exon 20 Insertion Mutations in Advanced Non-small Cell Lung Cancer in Korea. Cancer Res Treat, 51, 623-631 https://doi.org/10.4143/crt.2018.151 (2019).

\section{Figures}




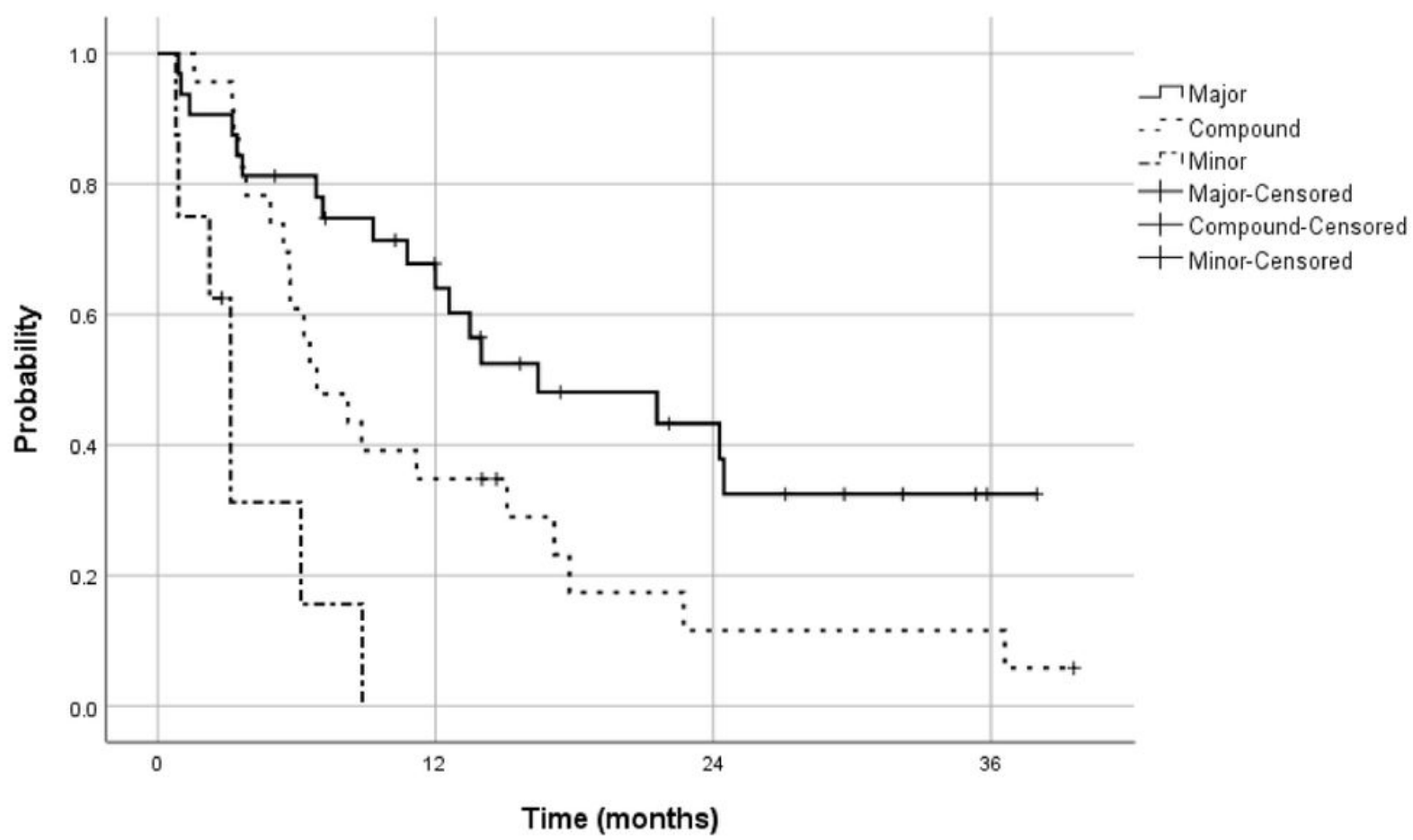

Figure 1

Time of treatment in patients with non-small cell lung cancer tumors harboring uncommon EGFR mutations $(p<0.001$ by log-rank test) 


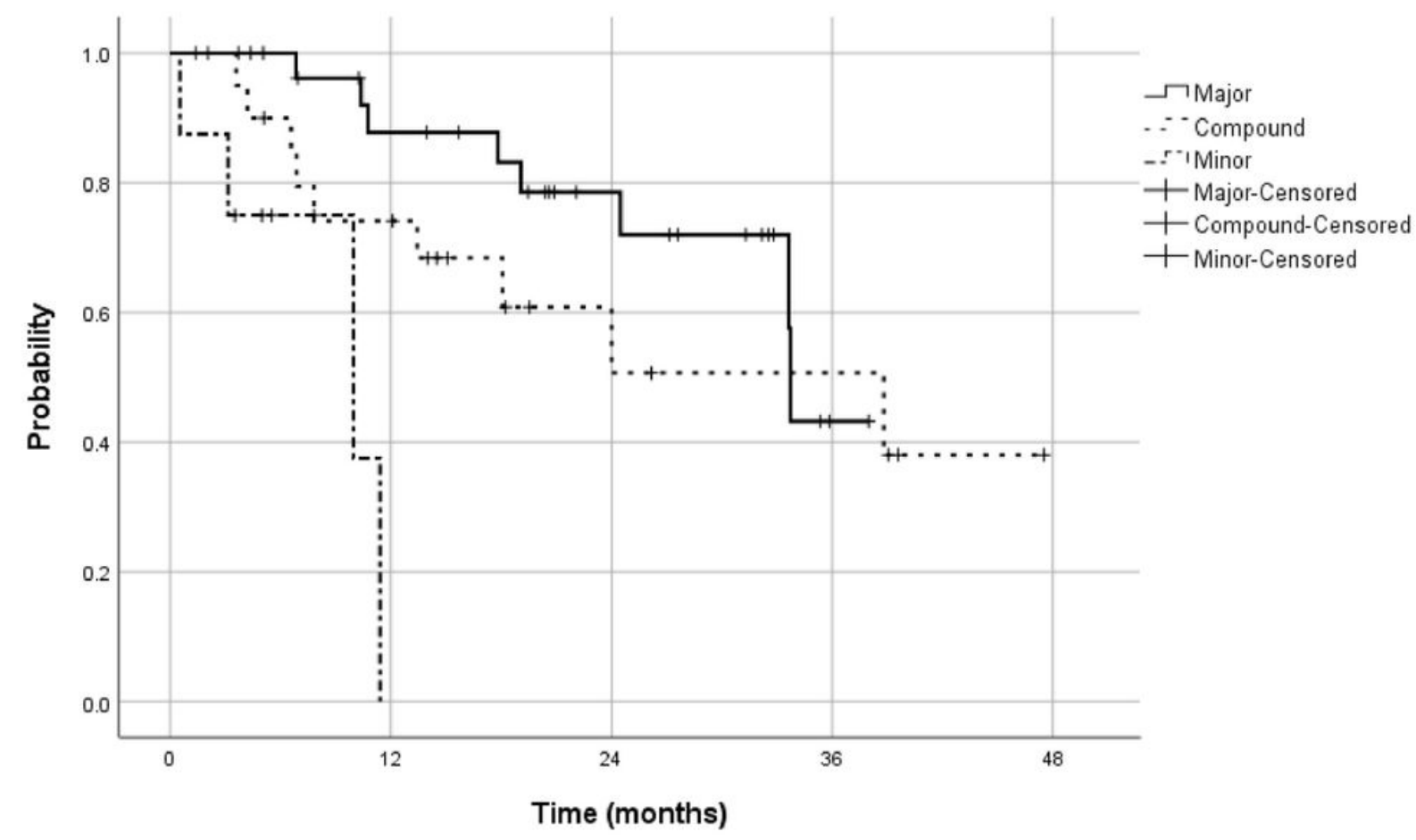

Figure 2

Overall survival in patients with non-small cell lung cancer tumors harboring uncommon EGFR mutations $(p=0.001$ by log-rank test $)$

(a)

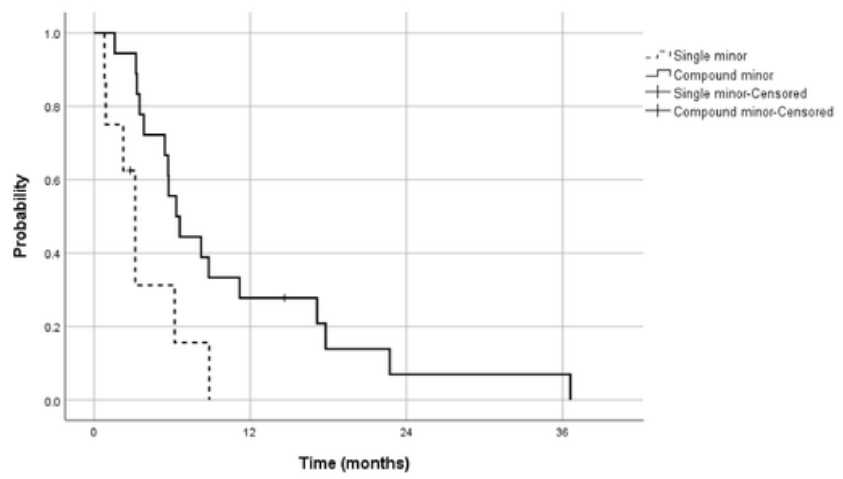

(b)

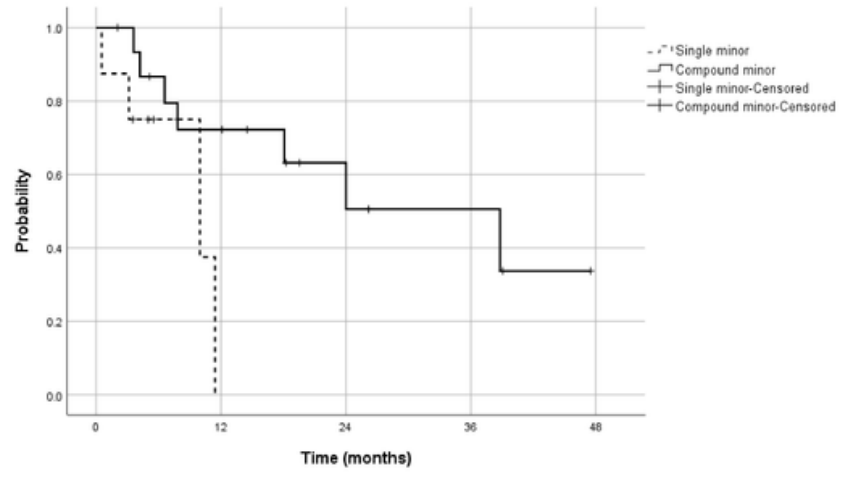


Figure 3

Time of treatment (a) and overall survival (b) in patients with minor uncommon EGFR mutations 